\title{
Treatment of Candida Glabrata With Micafungin: A Case Report and Brief Review of the Literature
}

\author{
Achyut Guleri $^{\mathrm{a}, \mathrm{b}}$, Daniel Gatt ${ }^{\mathrm{a}}$, Rashmi Sharma ${ }^{\mathrm{a}}$, Stephen Pettit ${ }^{\mathrm{a}}$
}

\begin{abstract}
We report the case of a patient with multiple Candida bloodstream infections post emergency laparotomy, Hartmann's procedure, abdominal closure and drainage. The patient also experienced recurrent central venous catheter Candida infections. The treatment of Candida tropicalis bloodstream infection with fluconazole and caspofungin was initially successful; however the patient consequently developed Candida glabrata bloodstream infection which did not respond to therapy with fluconazole or caspofungin. Although at the time of treatment of this patient, the C. glabrata strain was considered to be susceptible to caspofungin, the new clinical breakpoints recently published by the Clinical and Laboratory Standards Institute show that this strain was in fact resistant to caspofungin. The patient was successfully treated with micafungin and a line lock was used for the Hickman line. This case study is the first documented European case report of successful treatment of breakthrough C. glabrata blood stream infection with micafungin.
\end{abstract}

Keywords: Candida glabrata; Echinocandins; Treatment; Microbial sensitivity tests; Disease management

\section{Introduction}

The risk factors for invasive candidiasis include antibiotic use and duration, more than 4 days on intensive care or longer than 48 hours on mechanical ventilation, high APACHE II score, abdominal surgery, total parenteral nutrition (TPN), central venous catheter (CVC) in situ, and concomitant infection $[1,2]$. Around $60 \%$ of patients will have one or more of these risk factors [3]. The epidemiology of Candida bloodstream infection is changing, as most invasive fungal infec-

\footnotetext{
Manuscript accepted for publication April 25, 2013

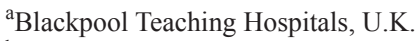

${ }^{\mathrm{b}}$ Corresponding author: Achyut Guleri, Blackpool Teaching Hospital, Whinney Heys Road; Blackpool; FY3 8NR, U.K.

Email: Dr.Guleri@bfwh.nhs.uk
}

doi: http://dx.doi.org/10.4021/jmc1237w tions previously caused by $C$. albicans, are now increasingly caused by C. glabrata, C. parapsilosis and C. krusei [3]. The change in the epidemiology of Candida bloodstream infection means that in vitro testing should include genus- and species-specific susceptibility testing to support clinical decisions.

The use of fluconazole prophylaxis has been associated with the increase in colonization and disease from azole resistant Candida strains $[4,5]$. It is reported that, in patients with more than 2 weeks azole prophylaxis, over $17 \%$ of $\mathrm{Can}$ dida spp. exhibited a reduced susceptibility to fluconazole [6]. Intrinsic low-level and acquired high-level azole resistance of C. glabrata has been postulated to have contributed to the emergence of this fungal pathogen in the intensive care unit [6]. As the use of the echinocandins has increased so has selection pressure, and sporadic reports of echinocandin resistance have begun to appear in the literature [7]. The molecular mechanisms of resistance to the echinocandins relate to amino acid substitutions within the FKS1 target gene. At the time of this patient's treatment, the Clinical Laboratory Standards Institute (CLSI) suggested a "susceptible only" breakpoint of $<2 \mu \mathrm{g} / \mathrm{mL}$ for Candida spp. for caspofungin, micafungin and anidulafungin [8]. However, infections involving isolates with target alterations in the FKS1 gene did not necessarily show minimum inhibitory concentration (MICs) above this breakpoint $[9,10]$. In addition, in vitro susceptibility testing does not take into account the effect of serum protein binding, which may influence the relative clinical efficacy of the echinocandins [10]. Thus, MIC and susceptibility testing are often poor predictors of clinical outcome in Candida bloodstream infection [11]. Recently, the CLSI have reset the breakpoints for Candida based on pharmacokinetic and pharmacodynamic modeling. Echinocandin breakpoints for Candida are now species specific and in the case of C. glabrata, different for micafungin than to caspofungin [12]. Similarly, the European Committee on Antimicrobial Susceptibility Testing (EUCAST) have also set drug-specific breakpoints for Candida for each of the echinocandin drugs, although due to inter laboratory variation in MIC ranges, the breakpoints for caspofungin against C. glabrata have not yet been established by EUCAST [13].

Here we report a case of breakthrough Candida blood- 
Table 1. Antifungal Agent Sensitivities to Seven Candida glabrata Isolates Taken From Case Study Patient

Isolate number (date of isolation in 2008)

\begin{tabular}{llllllll}
\cline { 2 - 7 } Antifungal agent & $\mathbf{1 8 , 7 7 6}$ & $\mathbf{1 8 , 7 7 9}$ & $\mathbf{1 8 , 7 7 8}$ & $\mathbf{1 8 , 7 7 7}$ & $\mathbf{1 8 , 7 7 5}$ & $\mathbf{1 8 , 7 7 4}$ & $\mathbf{1 8 , 7 7 3}$ \\
& $\mathbf{( 2 0 / 0 9 )}$ & $\mathbf{( 2 3 / 0 9 )}$ & $\mathbf{( 2 4 / 0 9 )}$ & $\mathbf{( 2 9 / 0 9 )}$ & $\mathbf{( 0 2 / 1 0 )}$ & $\mathbf{( 0 3 / 1 0 )}$ & $\mathbf{( 0 4 / 1 0 )}$ \\
\hline Flucytosine & SD & SD & SD & SD & SD & SD & SD \\
Amphotericin & 0.06 & 0.06 & 0.06 & 0.06 & 0.06 & 0.06 & 0.06 \\
Fluconazole & 8 & 8 & 8 & 8 & 8 & 8 & 8 \\
Itraconazole & 1 & 2 & 1 & 2 & 1 & 1 & 2 \\
Voriconazole & 0.25 & 0.25 & 0.25 & 0.125 & 0.125 & 0.5 & 0.25 \\
Caspofungin & 0.5 & 0.5 & 0.5 & 0.5 & 0.5 & 0.5 & 0.5 \\
Micafungin & $\leq 0.015$ & $\leq 0.015$ & $\leq 0.015$ & $\leq 0.015$ & $\leq 0.015$ & $\leq 0.015$ & $\leq 0.015$ \\
\hline
\end{tabular}

SD: sensitive by disc test.

stream infection in a 16-year-old male who had previously undergone an emergency laparotomy and had received repeated courses of fluconazole and caspofungin.

\section{Case Report}

In July 2008, a 16-year-old male weighing $50 \mathrm{~kg}$ was admitted to hospital with a previous history of constipation and a 4-day history of abdominal pain. Vital signs on admission included a respiratory rate of $32 \mathrm{bpm}$, blood pressure of $111 / 60 \mathrm{mmHg}$, and temperature of $38^{\circ} \mathrm{C}$. The patient was clinically dehydrated and peritonitic; a chest X-ray showed free intraperitoneal gas. Emergency laparotomy revealed a well-established perforation of the mid-sigmoid colon and extensive fecal contamination. A Hartmann's procedure was carried out, followed by thorough peritoneal lavage and primary abdominal closure. The patient was subsequently transferred to intensive care. In the post-operative period antibacterial prophylaxis with cefuroxime $(750 \mathrm{mg}$, TDS, days 1 to 6), metronidazole (500 $\mathrm{mg}$, TDS, days 1 to 6), piperacillin-tazobactam (4.5 g, TDS, Days 6 to 12), gentamicin (320 $\mathrm{mg}$, OD, days 7 to 13 ) and erythromycin (250 mg, TDS, days 7 to 12 ) was given.

Recovery from surgery was hampered by multiple infectious complications. On day 3 , the patient became pyrexial with a temperature of $39.2^{\circ} \mathrm{C}$. Staphylococcus epidermidis was cultured from blood cultures. The CVC was replaced on Day 9 from which Candida tropicalis was reported on day 12 ; in view of this, fluconazole $400 \mathrm{mg}$ OD was then started. Despite continued combined anti-microbial (meropenem 1 g, TDS days 13 to 18 ) and anti-fungal therapy, blood cultures remained positive for $\mathrm{S}$. epidermidis and $C$. tropicalis.

On day 12, the patient also developed a wound infection, and CT scan revealed a pelvic collection. Following dehiscence of the abdominal wound on day 16 which was treated with a VAC dressing, the patient developed a high volume enterocutaneous fistula on day 23 which drained several liters of effluent a day. The CVC was replaced on day 19, and again on day 26, when a Hickman line was introduced instead. The CVC tip culture returned as $C$. albicans positive. On day 35 , fluconazole was replaced with caspofungin ( 70 $\mathrm{mg}$ first dose and then $50 \mathrm{mg}$ OD) due to persistent fever and positive $C$. tropicalis blood culture. Two further line changes also followed. On day 46, the patient's fever finally abated and bloods returned without fungal-positive cultures. During caspofungin therapy the patient also received antimicrobial therapy with meropenem ( $1 \mathrm{~g}$, TDS, days 35 to 46), vancomycin ( $1 \mathrm{~g}, \mathrm{BD}$, days 41 to 45$)$ and gentamicin (240 mg, OD, days 32 to 38 ).

After a couple of days of being afebrile, the patient respiked a temperature, and blood cultures once again became positive for $S$. epidermidis. The CVC was changed for the sixth time and the patient received gentamicin therapy between days 48 and 51. On day 55, meropenem ( $1 \mathrm{~g}, \mathrm{TDS})$ and vancomycin (1 g, BD) were restarted and given until day 62 . On day 60, blood cultures returned positive for C. glabrata. Fluconazole $400 \mathrm{mg}$ OD was re-started and the CVC line changed again. Based on sensitivities (Table 1), the fluconazole therapy was replaced with caspofungin on day 66 . The ninth CVC was removed and a peripherally inserted central catheter (PICC) was inserted instead. Despite continued ca- 
spofungin therapy, the patient's condition did not improve. On day 84, the PICC line was replaced under cefuroxime, caspofungin plus voriconazole cover for 48-hours while micafungin availability was arranged by pharmacy. Taurolidine citrate 4\% line lock was used for the PICC. Micafungin 100 mg OD was started on day 86. On day 89, the PICC line had to be removed for non-infective reasons and a Hickman line inserted with continued taurolodine lock. Micafungin was given between days 86 to 101, and the patient had no further episodes of infection. The patient received TPN for the whole of this period.

The seven isolates of $C$. glabrata were tested in-house (fluconazole MIC $8 \mathrm{mg} / \mathrm{mL}$ ) and by the Mycology Regional Laboratory, Wythenshawe Hospital for their antifungal susceptibility. Azoles were tested using the European Committee on Antimicrobial Susceptibility Testing (EUCAST) method [14], amphotericin B using the Rex method [15] and caspofungin and micafungin using the CLSI M27-A3 method [8] (Table 1).

In summary, between days 11 and 46, the patient developed persistent Candida bloodstream infection ( 9 positive blood cultures) with $C$. tropicalis successfully treated with caspofungin. However, between days 62 and 77, the patient again developed persistent Candida bloodstream infection (8 positive blood cultures) this time with C. glabrata that did not respond to caspofungin but which responded to micafungin and concomitant lock therapy with taurolidine 4\% citrate.

After an in-patient stay in excess of three months, the patient was transferred to a specialist centre. He remained afebrile with the same Hickman line and had no further infectious complications. The patient developed inevitable intestinal failure and remained on home parenteral nutrition. In the following months, he went on to have reconstructive bowel surgery in the form of closure of the abdominal wall defects and resection of small bowel fistulae; the double barreled jejunostomy which was created has since been closed and the patient has been left with a colostomy.

\section{Discussion}

Literature reports breakthrough Candida bloodstream infection with in vitro-susceptible Candida strains during empiric therapy with fluconazole [16]. We believe that this case study represents the first documented European case of successful treatment of breakthrough C. glabrata infection with micafungin following unsuccessful treatment with caspofungin. The condition of the patient improved, and fever resolved, after the third Hickman line change and initiation of treatment with micafungin. The therapeutic failure of caspofungin against a then - considered susceptible $C$. glabrata strain is a matter of concern and raises a number of issues.

The relevance of MIC testing for the echinocandins remains unclear [9]. The lack of correlation between elevated
MICs and clinical outcome with other antifungals, namely the azoles, was first reported in the literature in 1995 [17]. In this study 232 isolates were collected during a clinical trial of fluconazole and amphotericin B for the treatment of Candida bloodstream infection in non-neutropenic patients. Elevated MICs did not correlate with treatment failure in this study, indeed treatment outcome was successful in four patients despite MICs of $>32 \mu \mathrm{g} / \mathrm{mL}$. This study group suggested that host factors (such as failure to exchange CVCs) may be more important than MIC in predicting outcome. Uncertain correlation between higher MIC and successful clinical outcome with $C$. parapsilosis has also been reported in the literature [18]. To investigate the relationship between $\mathrm{MIC}$ and outcome, a retrospective analysis of isolates from the caspofungin clinical trial database was undertaken that found no correlation between lower MICs and favorable outcome; indeed, patients with higher MICs $(>2 \mu \mathrm{g} / \mathrm{mL})$ had better outcomes [19]. These studies highlight the difficulty of interpreting antifungal MIC results in the clinical setting. Although Etests have been shown to produce a wider distribution of MICs than either culture or broth microdilution, no one test has been shown to generate results that significantly correlate with success or failure [20].

Guidelines recommend that in the presence of elevated MICs to the echinocandins; organism identification and susceptibility testing should be confirmed using a CLSI reference dilution method [21]. The CLSI has now recommended species specific echinocandin breakpoints for Candida species. For C. glabrata, the susceptible breakpoint for micafungin is lower $(0.012 \mu \mathrm{g} / \mathrm{mL})$ than for caspofungin or anidulafungin $(0.06 \mathrm{mg} / \mathrm{mL})$. Looking back at the susceptibilities retrospectively, the $C$. glabrata strain in this patient was resistant $(0.5 \mu \mathrm{g} / \mathrm{mL})$ to caspofungin but was susceptible $(\leq 0.015 \mu \mathrm{g} / \mathrm{mL})$ to micafungin [12]. Pharmacokinetic/ pharmacodynamic modeling techniques have provided more accurate information for current and future decision-support analysis.

The points raised in this discussion highlight how the choice of a suitable anti-fungal drug is of paramount importance in the treatment of patients with Candida bloodstream infections. Although amphotericin B, based on its broad spectrum of activity against Candida spp., could be argued to have been an option to consider in this patient, both the Infections Diseases Society of America (IDSA) and European Society for Clinical Microbiology and Infectious Diseases (ESCMID) guidelines for the management of candidiasis [2, 22] recommend an echinocandin in preference over amphotericin B for non-neutropenic patients with Candida bloodstream infections, due to concerns of amphotericin B's toxicity. The high degree of well-documented cross-resistance within the azole class $[2,22]$ would have also limited the usefulness of alternative azole agents such as voriconazole for this particular patient following treatment failure with fluconazole. 
Other issues in the management of patients with Candida bloodstream infection include host factors, the role of biofilms and the use of line locks. This case of a patient with many of the known risk factors for Candida bloodstream infection, including TPN, abdominal surgery and the use of broad spectrum antibiotics, clearly illustrates the difficulty of managing such patients in clinical practice. Interestingly, in this case there were no further breakthrough Candida bloodstream infections after the Taurolock ${ }^{\mathrm{TM}}$ (TauroPharm $\mathrm{GmbH}$, Germany) was introduced as a line lock and the switch to micafungin as the antifungal agent. Micafungin, has lower MICs than other members of the class against $C$. glabrata (Table 1), which despite the lack of robust evidence for a relationship between MIC and outcome, may have been a contributing factor in the resolution of the multiple Candida bloodstream infections in this patient. Further clinical studies are needed to assess the relevance of MIC to outcome and the influence of other management strategies such as lock therapy, in the complex management of patients with multiple co-morbidities and invasive fungal infections.

\section{Acknowledgement and Funding}

The contribution of Ms. Caroline Moore, Deputy Director Mycology Reference Laboratory, Wythenshawe Hospital, Manchester; Dr CPK Subudhi and Dr R Kalaiselvan, Salford Royal NHS Foundation Trust in the preparation of this case study are acknowledged. The medical writer, Ms Tracy J. Taylor and publication fee was funded by Astellas Pharma Limited.

\section{Conflicts of Interest}

Dr Guleri has received payments from Astellas Pharma Limited as an advisory board member and has received conference sponsorship.

Dr Sharma, Mr Pettit and Dr Gatt: none.

Ms Taylor is a former employee of Astellas Pharma Europe Limited and her contribution as medical writer for this manuscript was funded by Astellas Pharma Limited.

\section{Declaration}

The study was presented in part at the European Congress of Clinical Microbiology and Infectious Diseases, Helsinki, Finland 16 - 19th May 2009, Abstract 1308.

\section{References}

1. Ostrosky-Zeichner L, Sable C, Sobel J, Alexander BD,
Donowitz G, Kan V, Kauffman CA, et al. Multicenter retrospective development and validation of a clinical prediction rule for nosocomial invasive candidiasis in the intensive care setting. Eur J Clin Microbiol Infect Dis. 2007;26(4):271-276.

2. Pappas PG, Kauffman CA, Andes D, Benjamin DK, Jr., Calandra TF, Edwards JE, Jr., Filler SG, et al. Clinical practice guidelines for the management of candidiasis: 2009 update by the Infectious Diseases Society of America. Clin Infect Dis. 2009;48(5):503-535.

3. Richardson M, Lass-Florl C. Changing epidemiology of systemic fungal infections. Clin Microbiol Infect. 2008;14(Suppl 4):5-24.

4. Marr KA, Seidel K, White TC, Bowden RA. Candidemia in allogeneic blood and marrow transplant recipients: evolution of risk factors after the adoption of prophylactic fluconazole. J Infect Dis. 2000;181(1):309-316.

5. Charlier C, Hart E, Lefort A, Ribaud P, Dromer F, Denning DW, Lortholary O. Fluconazole for the management of invasive candidiasis: where do we stand after 15 years? J Antimicrob Chemother. 2006;57(3):384-410.

6. Leroy O, Gangneux JP, Montravers P, Mira JP, Gouin F, Sollet JP, Carlet J, et al. Epidemiology, management, and risk factors for death of invasive Candida infections in critical care: a multicenter, prospective, observational study in France (2005-2006). Crit Care Med. 2009;37(5):1612-1618.

7. Arendrup MC, Perkhofer S, Howard SJ, Garcia-Effron G, Vishukumar A, Perlin D, Lass-Florl C. Establishing in vitro-in vivo correlations for Aspergillus fumigatus: the challenge of azoles versus echinocandins. Antimicrob Agents Chemother. 2008;52(10):3504-3511.

8. CLSI. Zone Diameter Interpretive Standards, Corresponding Minimal Inhibitory Concentration (MIC) Interpretive Breakpoints, and Quality Control Limits for Antifungal Disk Diffusion Susceptibility Testing of Yeasts; Informational Supplement. CLSI document M44-S2. Wayne, PA: Clinical and Laboratory Standards Institute; 2009.

9. Arendrup MC, Garcia-Effron G, Buzina W, Mortensen KL, Reiter N, Lundin C, Jensen HE, et al. Breakthrough Aspergillus fumigatus and Candida albicans double infection during caspofungin treatment: laboratory characteristics and implication for susceptibility testing. Antimicrob Agents Chemother. 2009;53(3):1185-1193.

10. Garcia-Effron G, Park S, Perlin DS. Correlating echinocandin MIC and kinetic inhibition of fks1 mutant glucan synthases for Candida albicans: implications for interpretive breakpoints. Antimicrob Agents Chemother. 2009;53(1):112-122.

11. Krause DS, Reinhardt J, Vazquez JA, Reboli A, Goldstein BP, Wible M, Henkel T. Phase 2, randomized, dose-ranging study evaluating the safety and efficacy of anidulafungin in invasive candidiasis and candidemia. 
Antimicrob Agents Chemother. 2004;48(6):2021-2024.

12. Pfaller MA, Diekema DJ, Andes D, Arendrup MC, Brown SD, Lockhart SR, Motyl M, et al. Clinical breakpoints for the echinocandins and Candida revisited: integration of molecular, clinical, and microbiological data to arrive at species-specific interpretive criteria. Drug Resist Updat. 2011;14(3):164-176.

13. European Committee on Antimicrobial Susceptibility Testing. Antifungal Agents. Breakpoint tables for interpretation of MICs. Version 6.0, 1 March 2013. http:// www.eucast.org.

14. Cuenca-Estrella M, Arendrup MC, Chryssanthou E, Dannaoui E, Lass-Florl C, Sandven P, Velegraki A, et al. Multicentre determination of quality control strains and quality control ranges for antifungal susceptibility testing of yeasts and filamentous fungi using the methods of the Antifungal Susceptibility Testing Subcommittee of the European Committee on Antimicrobial Susceptibility Testing (AFST-EUCAST). Clin Microbiol Infect. 2007;13(10):1018-1022.

15. Rex JH, Cooper CR, Jr., Merz WG, Galgiani JN, Anaissie EJ. Detection of amphotericin B-resistant Candida isolates in a broth-based system. Antimicrob Agents Chemother. 1995;39(4):906-909.

16. Kovacicova G, Mateicka F, Hanzen J, Liskova A, Sabo A, Szovenyova Z, Chmelik B, et al. Breakthrough candidaemias during empirical therapy with fluconazole in non-cancer and non-HIV adults caused by in vitro-susceptible Candida spp.: report of 33 cases. Scand J Infect Dis. 2001;33(10):749-751.

17. Rex JH, Pfaller MA, Barry AL, Nelson PW, Webb CD. Antifungal susceptibility testing of isolates from a ran- domized, multicenter trial of fluconazole versus amphotericin B as treatment of nonneutropenic patients with candidemia. NIAID Mycoses Study Group and the Candidemia Study Group. Antimicrob Agents Chemother. 1995;39(1):40-44.

18. Brito LR, Guimaraes T, Nucci M, Rosas RC, Paula Almeida L, Da Matta DA, Colombo AL. Clinical and microbiological aspects of candidemia due to Candida parapsilosis in Brazilian tertiary care hospitals. Med Mycol. 2006;44(3):261-266.

19. Kartsonis N, Killar J, Mixson L, Hoe CM, Sable C, Bartizal $\mathrm{K}$, Motyl M. Caspofungin susceptibility testing of isolates from patients with esophageal candidiasis or invasive candidiasis: relationship of MIC to treatment outcome. Antimicrob Agents Chemother. 2005;49(9):3616-3623.

20. Park BJ, Arthington-Skaggs BA, Hajjeh RA, Iqbal N, Ciblak MA, Lee-Yang W, Hairston MD, et al. Evaluation of amphotericin B interpretive breakpoints for Candida bloodstream isolates by correlation with therapeutic outcome. Antimicrob Agents Chemother. 2006;50(4):12871292.

21. Pfaller MA, Diekema DJ, Ostrosky-Zeichner L, Rex JH, Alexander BD, Andes D, Brown SD, et al. Correlation of MIC with outcome for Candida species tested against caspofungin, anidulafungin, and micafungin: analysis and proposal for interpretive MIC breakpoints. J Clin Microbiol. 2008;46(8):2620-2629.

22. Cornely OA, Bassetti M, Calandra T, Garbino J, Kullberg BJ, Lortholary O, Meersseman W, et al. ESCMID* guideline for the diagnosis and management of Candida diseases 2012: non-neutropenic adult patients. Clin Microbiol Infect. 2012;18(Suppl 7):19-37. 